\title{
Correction to: Investigation of novel variations of ORAl1 gene and their association with Kawasaki disease
}

\author{
Kyaw Thiha ${ }^{1} \cdot$ Yoichi Mashimo $^{1} \cdot$ Hiroyuki Suzuki $^{2} \cdot$ Hiromichi Hamada $^{3} \cdot$ Akira Hata $^{1} \cdot$ Toshiro Hara $^{4}$. \\ Toshihiro Tanaka $\circledast^{5} \cdot$ Kaoru Ito ${ }^{6}$ Y Yoshihiro Onouchi ${ }^{1,6} \cdot$ Japan Kawasaki Disease Genome Consortium
}

Published online: 31 July 2019

(c) The Author(s), under exclusive licence to The Japan Society of Human Genetics 2019

\section{Correction to: Journal of Human Genetics https://doi.org/10.1038/s10038-019-0588-2 published online 11 March 2019}

Affiliations of the Japan Kawasaki Disease Genome Consortium that are listed in Acknowledgements were incorrect.

Corrected affiliations are as follows.

Japan Kawasaki Disease Genome Consortium Hiroshi Masuda ${ }^{7}$, Tohru Kobayashi $^{7}$, Toshiaki Jibiki ${ }^{8}$, Taro Yamazaki ${ }^{9}$, Yoshiaki Ohkuma ${ }^{10}$, Maya Fujiwara ${ }^{11}$, Tomio Kobayashi ${ }^{12}$, Harumitsu Takeuchi ${ }^{13}$, Kazunobu Ouchi ${ }^{14}$, Yumi Mizuno ${ }^{15}$, Shigeto Fuse ${ }^{16}$, Nobuhiro Fukazawa $^{17}$, Tsutomu Saji ${ }^{18}$, Shinichi Takatsuki ${ }^{18}$, Kenichiro Nishimura $^{19}$, Hiromichi Hamada ${ }^{20}$, Ryuji Fukazawa ${ }^{21}$, Hideko Nishumura $^{22}$, Kentaro $\mathrm{Aso}^{23}$, Tomoyo Matsubara ${ }^{24}$, Takahisa Mizuno $^{25}$, Satoru Iwashima ${ }^{26}$, Mamoru Ayusawa ${ }^{27}$, Kazuyuki Ikeda ${ }^{28}$, Takashi Kosuda ${ }^{29}$, Kunio Hashimoto ${ }^{30}$, Kunio Hirasawa ${ }^{31}$, Masaru Miura $^{32}$, Junpei Somura ${ }^{33}$, Emi Toba ${ }^{34}$, Keiichi Hirono ${ }^{35}$, Yuichi Nomura $^{36}$, Hirokazu Arakawa ${ }^{37}$, Shouhei Ogata ${ }^{38}$, Hiroki Kajino ${ }^{39}$, Seiji Kawamura ${ }^{40}$, Hayato Aoyagi ${ }^{41}$, Hiroshi Suzuki ${ }^{42}$, Kouta Ichinose $^{43}$, Ayako Shimozono ${ }^{44}$, Yoshiaki Kato ${ }^{45}$, Masamune Higashikawa $^{46}$, Youichi Kawamura ${ }^{47}$, Masahiro Misawa ${ }^{48}$, Noriko $\mathrm{Nagai}^{49}$, Taichi Kato ${ }^{50}$, Dan Nagata ${ }^{51}$, Akiko Okamoto ${ }^{52}$, Hiroyuki Suzuki $^{53}$, Masahiko Kishiro ${ }^{54}$, Junko Shiono ${ }^{55}$, Kouji Higashi ${ }^{56}$, Naoki Yokoyama $^{57}$, Ryota Ebata ${ }^{58}$, Yoshihiro Onouchi ${ }^{59}$.

Yoshihiro Onouchi

onouchy@chiba-u.jp

1 Department of Public Health, Chiba University Graduate School of Medicine, Chiba, Chiba, Japan

2 Department of Pediatrics, Wakayama Medical University, Wakayama, Wakayama, Japan

3 Department of Pediatrics, Tokyo Women's Medical University, Yachiyo Medical Center, Yachiyo, Chiba, Japan

4 Fukuoka Children's Hospital and Medical Center for Infectious Diseases, Fukuoka, Japan

5 Department of Human Genetics and Disease Diversity, Tokyo Medical and Dental University, Tokyo, Japan

6 Laboratory for Cardiovascular Diseases, RIKEN Center for Integrative Medical Sciences, Yokohama, Kanagawa, Japan
${ }^{7}$ National Center for Child Health and Development, Tokyo, Japan; ${ }^{8}$ Chiba Kaihin Municipal Hospital, Chiba, Japan; ${ }^{9}$ Saitama Medical University, Moroyama, Japan; ${ }^{10}$ National Center for Global Health and Medicine, Tokyo, Japan; ${ }^{11}$ Kawakita General Hospital, Tokyo, Japan; ${ }^{12}$ Gunma Children's Medical Center, Shibukawa, Japan; ${ }^{13}$ Kiryu Kosei General Hospital, Kiryu, Japan; ${ }^{14}$ Kawasaki Medical School, Kurashiki, Japan; ${ }^{15}$ Fukuoka Children's Hospital, Fukuoka, Japan; ${ }^{16}$ NTT Sapporo Medical Center, Sapporo, Japan; ${ }^{17}$ Fujioka General Hospital, Fujioka, Japan; ${ }^{18}$ Toho University Medical Center Omori Hospital, Tokyo, Japan; ${ }^{19}$ Yokohama City University Hospital, Yokohama, Japan; ${ }^{20}$ Tokyo Women's Medical University Yachiyo Medical Center, Yachiyo, Japan; ${ }^{21}$ Nippon Medical School, Tokyo, Japan; ${ }^{22}$ Tone Chuo Hospital, Numata, Japan; ${ }^{23}$ St. Marianna University School of Medicine, Kawasaki, Japan; ${ }^{24}$ Dokkyo Medical University Saitama Medical Center, Koshigaya, Japan; ${ }^{25}$ Gunma Chuo Hospital, Maebashi, Japan; ${ }^{26}$ Hamamatsu University School of Medicine, Hamamatsu, Japan; ${ }^{27}$ Nihon University School of Medicine, Tokyo, Japan; ${ }^{28}$ Graduate School of Medical Science, Kyoto Prefectural University of Medicine, Kyoto, Japan; ${ }^{29}$ Tomioka General Hospital, Tomioka, Japan; ${ }^{30}$ Nagasaki University Graduate School of Biomedical Sciences, Nagasaki, Japan; ${ }^{31}$ Fukaya Red Cross Hospital, Fukaya, Japan; ${ }^{32}$ Tokyo Metropolitan Children's Medical Center, Tokyo, Japan; ${ }^{33}$ Shiga University of Medical Science, Otsu, Japan; ${ }^{34}$ Minamitama Hospital, Hachioji, Japan; ${ }^{35}$ Graduate School of Medicine, University of Toyama, Toyama, Japan; ${ }^{36}$ Kagoshima University School of Medicine, Kagoshima, Japan; ${ }^{37}$ Gunma University Graduate School of Medicine, Maebashi, Japan; ${ }^{38}$ Kitasato University Hospital, Sagamihara, Japan; ${ }^{39}$ Abashiri-Kosei General Hospital, Abashiri, Japan; ${ }^{40}$ Kameda General Hospital, Kamogawa, Japan; ${ }^{41}$ Obihiro Kyokai Hospital, Obihiro, Japan; ${ }^{42}$ Niigata University Graduate School of Medicine, Niigata, Japan; ${ }^{43}$ Aomori City Hospital, Aomori, Japan; ${ }^{44}$ Tsuchiya General Hospital, Hiroshima, Japan; ${ }^{45}$ University of Tsukuba, Tsukuba, Japan; ${ }^{4}$ Ise Red Cross Hospital, Ise, Japan; ${ }^{47}$ National Defense Medical College, Tokorozawa, Japan; ${ }^{48}$ Tokyo Metropolitan Bokutoh Hospital, Tokyo, Japan; ${ }^{49}$ Okazaki City Hospoital, Okazaki, Japan; ${ }^{50}$ Nagoya University Hospital, Nagoya, Japan; ${ }^{51}$ Kyushu University Graduate School of Medicine, Fukuoka, Japan; ${ }^{52}$ Japanese Red Cross Kyoto Daiichi Hospital, Kyoto, Japan; ${ }^{53}$ Wakayama Medical University, Wakayama, Japan; ${ }^{54}$ Juntendo University, Graduate School of Medicine, Tokyo, Japan; ${ }^{55}$ Ibaraki Children's Hospital, Mito, Japan; ${ }^{56}$ Chiba Children's Hospital, Chiba, Japan; ${ }^{57}$ Akashi Medical Center, Akashi, Japan; ${ }^{58}$ Chiba University, Chiba, Japan; ${ }^{59}$ RIKEN, Yokohama, Japan 